\title{
Les restructurations militaires en Lorraine : enjeux d'aménagement à différentes échelles
}

Military restructuring in Lorraine: planning stakes at different scales

\section{Pierre Ginet}

\section{OpenEdition}

\section{Journals}

Édition électronique

URL : http://journals.openedition.org/rge/3242

DOI : $10.4000 /$ rge.3242

ISSN : 2108-6478

Éditeur

Association des géographes de l'Est

Édition imprimée

Date de publication : 30 mai 2011

ISSN : 0035-3213

\section{Référence électronique}

Pierre Ginet, "Les restructurations militaires en Lorraine : enjeux d'aménagement à différentes

échelles », Revue Géographique de l'Est [En ligne], vol. 51 / 1-2 | 2011, mis en ligne le 15 décembre 2011, consulté le 08 septembre 2020. URL : http://journals.openedition.org/rge/3242 ; DOI : https://doi.org/ 10.4000/rge.3242

Ce document a été généré automatiquement le 8 septembre 2020

Tous droits réservés 


\title{
Les restructurations militaires en Lorraine : enjeux d'aménagement à différentes échelles
}

Military restructuring in Lorraine: planning stakes at different scales

\author{
Pierre Ginet
}

\section{NOTE DE L'AUTEUR}

Article achevé de rédiger le 1er janvier 2011

\section{Introduction}

1 L'évolution des menaces pesant sur la France et la Révision Générale des Politiques Publiques ont conduit le Président de la République à commanditer la rédaction d'un Livre Blanc proposant une nouvelle configuration des Armées (Commission du Livre blanc sur la défense et la sécurité nationale, 2008). La suppression de 54.000 postes doit ramener à l'horizon 2014 l'effectif du Ministère de la Défense à 225.000 personnels civils et militaires. La réforme des Armées provoquera la disparition de plusieurs unités dont la présence contribue à la vie et à l'identité de la région Lorraine (Conseil Economique et Social de Lorraine, 2008). Terre déchirée après la guerre de 1870, martyrisée lors de la première guerre mondiale, éprouvée par la seconde puis malmenée par les mutations industrielles de la fin du XXème siècle, la Lorraine est de nouveau sacrifiée sur l'autel de l'intérêt national. Un premier niveau d'analyse nous conduit à nous interroger sur l'impact de la réforme sur la Lorraine et ses territoires et sur les dispositifs mis par l'Etat à la disposition des acteurs politiques locaux pour développer des démarches compensatoires. Des initiatives qui se sont d'ailleurs multipliées et ont contribué à faire émerger des projets urbains qui semblent, si on les examine au cas par cas, jouer leur rôle de relance 
territoriale. Le rôle du géographe ne peut toutefois pas se réduire à la simple addition de diagnostics territoriaux localisés. Sa capacité à formuler des hypothèses et des scénarios de développement impliquant l'articulation des différentes échelles spatiales et temporelles, nous amènera ensuite à nous interroger sur les enjeux soulevés par la réforme des Armées en termes d'aménagement du territoire national. En effet, le Livre Blanc de 2008 ne constitue-t-il pas également pour l'Etat un élément de mise en œuvre de sa vision de l'organisation future du territoire national ? Quelle serait alors la place de la Lorraine dans cette configuration? Dans quelle mesure le tissu géopolitique lorrain, au travers de ses particularités organisationnelles et fonctionnelles, contribue-t-il au succès de cette stratégie?

\section{I - Conséquences directes de la réforme des Armées sur les territoires lorrains : une réelle capacité de rebond?}

2 Les démarches compensatoires, analysées une par une laissent penser à un succès, même si certaines insuffisances se font jour.

\section{A - Géographie aréolaire de la restructuration issue de la réforme des Armées}

3 La Moselle et l'agglomération messine payent le plus lourd tribut à la réforme des Armées. En 2006, 8.000 personnes travaillaient pour le Ministère de la Défense, soit 6 $\%$ de la population active de la ville de Metz. Après la réorganisation ce chiffre se réduira à 4.000 ! Une restructuration qui entraînera le départ de l'agglomération de 12.000 personnes et affectera les logements, les écoles par la fermeture de classes, le tissu associatif et l'économie locale (entreprises travaillant avec le Ministère de la Défense).

Metz et la Moselle, cœur de cible de la réforme des Armées

4 Une demi-douzaine de sites sont concernés dans l'agglomération messine (Kubiak, Serre, 2010): le Quartier Lizé, dont les neuf hectares font l'objet d'un projet d'accueil de la Direction Régionale de l'Environnement, de l'Aménagement et du Logement de Lorraine; l'hôtel du Délégué Militaire Départemental et l'Hôtel Poncelet, qui quittent également le domaine militaire; le site de la Direction régional du Matériel, occupé par le Ministère de la Défense depuis $1951\left(20.000 \mathrm{~m}^{2}\right.$ dont 3.000 de surface bâtie) et réinvesti depuis mai 2010 par le Centre d'Appel Interministériel de Metz.

5 Dans l'agglomération de Metz cette fois (Doc. $\mathrm{n}^{\circ} 1$ ), à Châtel-Saint-Germain, les bâtiments du 1er RMed, Quartier Serret, feront l'objet d'une réaffectation courant 2011, à l'occasion du déménagement du régiment. Cette caserne se compose d'un ensemble de bâtiments rénovés récemment et par conséquent non cessibles à la commune pour l'euro symbolique (cf. chapitre B). L'éducation nationale a projeté d'y réaliser un internat d'excellence sur le modèle de Sourdun. Le Ministère de l'Intérieur est également intéressé par les locaux, afin d'y installer des CRS et une école de formation. Les deux occupants partageront certains équipements 
(installations sportives, amphithéâtres, chaînes de restauration) et ce, dès le départ du 1e RMed, permettant d'éviter la phase de gardiennage.

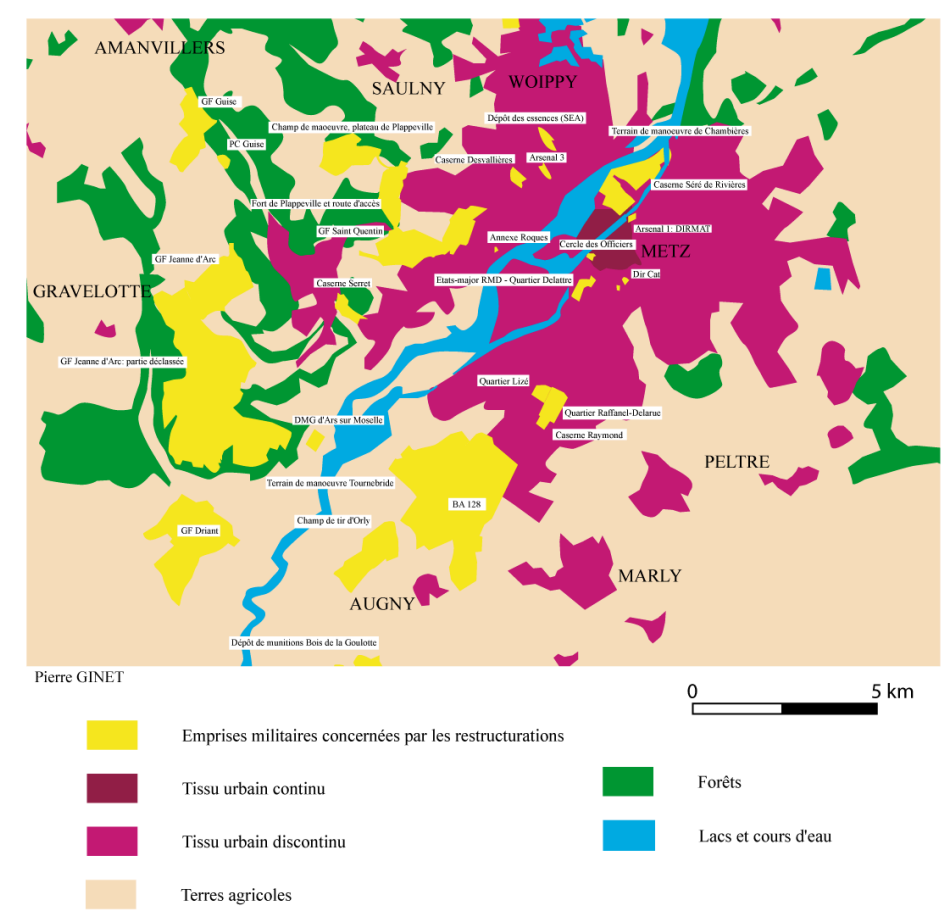

Document $n^{\circ} 1$. Espaces concernés par les restructurations militaires dans l'agglomération de Metz.

6 Enfin le site Séré de Rivières et la Cartoucherie Chambière devaient également quitter le domaine militaire. Mais alors que l'aliénation de ces sites paraissait inévitable, le président de la République annonce à l'automne 2009, l'arrivée du 3e Régiment de Hussards d'Immendingen (Allemagne) avant 2012 pour y remplacer le régiment du génie.

7 Hormis ces sites relevant de l'Armée de Terre, l'emprise la plus importante en superficie est celle de la Base Aérienne 128, située sur le territoire de la commune d'Augny (Doc. n²), qui elle, sera libérée en 2012. Elle fait l'objet d'un projet d'écocité (Ecocité128) dans le cadre d'affectations financières de l'Etat consécutives au Grenelle de l'environnement. Un projet dont les contours ne sont pas encore précisément établis et dont la livraison s'inscrit dans une perspective probable de plusieurs décennies. 


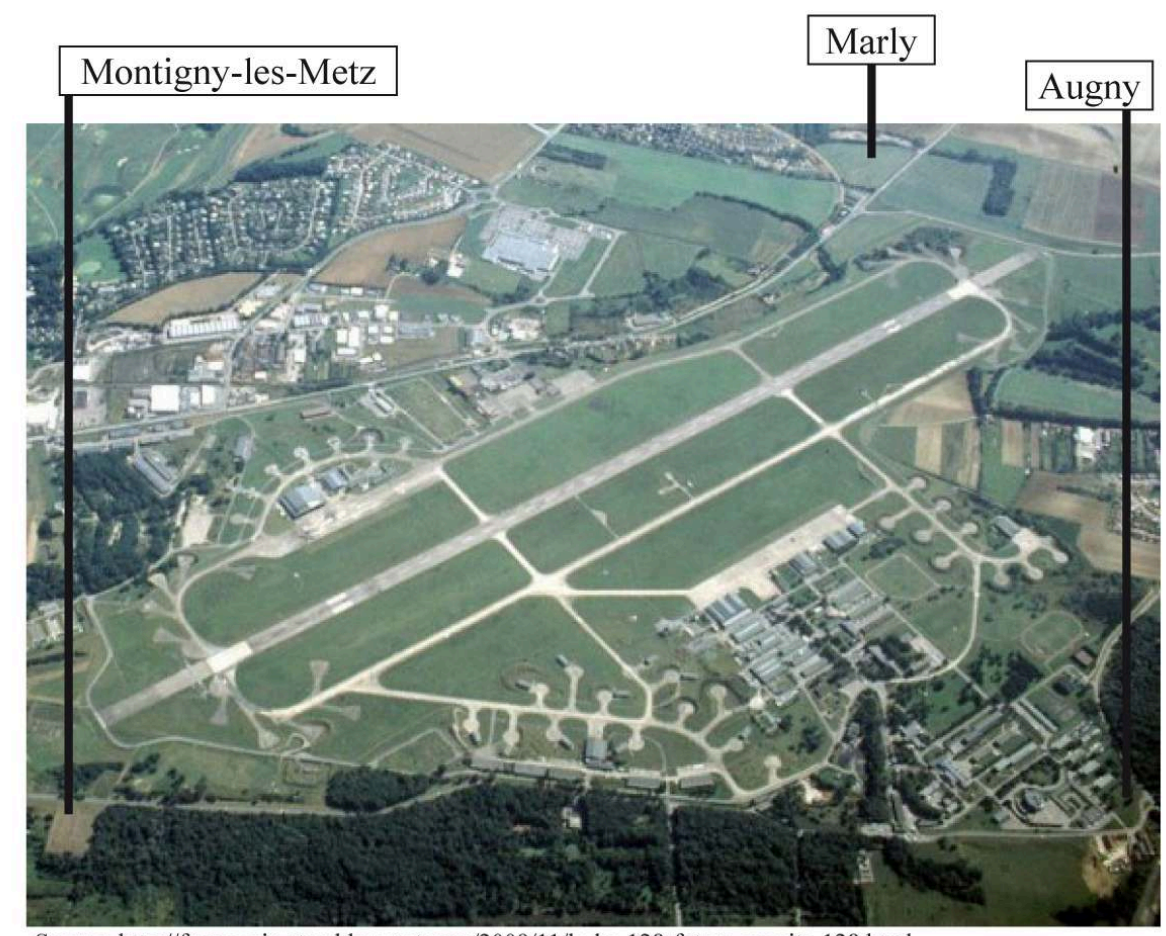

Source: http://france-air-otan.blogspot.com/2009/11/la-ba-128-future-ecocite-128.html

Document $n^{\circ} 2$. Base aérienne 128

\section{Les agglomérations mosellanes de Bitche et de Dieuze, également touchées}

8 La fermeture de la caserne du 57e RA présent depuis 1977 à Bitche (Doc. n³) a durement affecté cette ville de 5.700 habitants, déjà touchée en 1994 par la perte d'un escadron. Concernant 1.257 militaires, 120 civils et même 2.500 personnes si l'on compte les familles, c'est une grande partie de la ville qui va partir. En janvier 2009, l'annonce de l'arrivée du 16e BC rassure la population. Depuis, le 57e RA a quitté Bitche et le 16e BC l'a remplacé.

9 Comme à Metz, l'Etat, après avoir fait deux pas en arrière, en fait un en avant. Désormais, la Communauté de communes de Bitche souhaite acquérir les $2.300 \mathrm{~m}^{2} \mathrm{de}$ la caserne Aynie, bâtiment construit en 1897, pour y héberger des équipements publics. La création d'un chemin patrimonial est envisagée. D'autres projets ont par contre avorté: un parc national de feuillus, sur cinquante hectares du camp de Bitche, l'aménagement d'un espace récréatif près de l'étang d'Haspelschied (Doc. $\mathrm{n}^{\circ} 4$ ) et l'installation d'une centrale photovoltaïque. On notera qu'en raison de l'arrivée du 16e BC, Bitche n'est pas éligible au Contrat de Redynamisation de Site de Défense (CRSD, cf. chapitre B) mais au Plan Local de Redynamisation (PLR), une partie des financements de ce dispositif, ventilés au niveau départemental, ayant déjà été utilisée pour la création d'un réseau Internet à haut débit.

10 Le départ du 13e RDP en 2011, premier employeur de Dieuze avec 930 militaires et civils, provoquera la disparition du quart de la population de Dieuze avec à la clef une baisse de $10 \%$ des élèves scolarisés. Cela conduira probablement à une réorganisation scolaire voire à la suppression de classes ou même d'établissements (Kubiak, Serre, 2009). Signalons la volonté du maire de Dieuze de récupérer les terrains d'entrainement du 13e RDP de Donjeux, Guéblange-lès-Dieuze et Viviers, 
éligibles à l'euro symbolique. Mais la commune voisine de Delme est également intéressée par le site de Donjeux et Viviers pour l'installation de panneaux photovoltaïques (Barbier, 2010). Le CRSD et ses 12 millions d'euros, devraient permettre de financer un projet haut débit à hauteur de quatre millions d'euros. D'autres projets ont vu le jour, comme la création d'un hôtel aux Salines Royales ou la transformation d'un grenier de sel en complexe multiculturel. Une petite partie de l'enveloppe globale sera utilisée pour aménager le centre ville de Château-Salins. L'aide du Fond de Restructuration de la Défense (FReD) fournira un appui aux entreprises souhaitant créer des emplois, grâce à une enveloppe de plus de six millions d'euros.

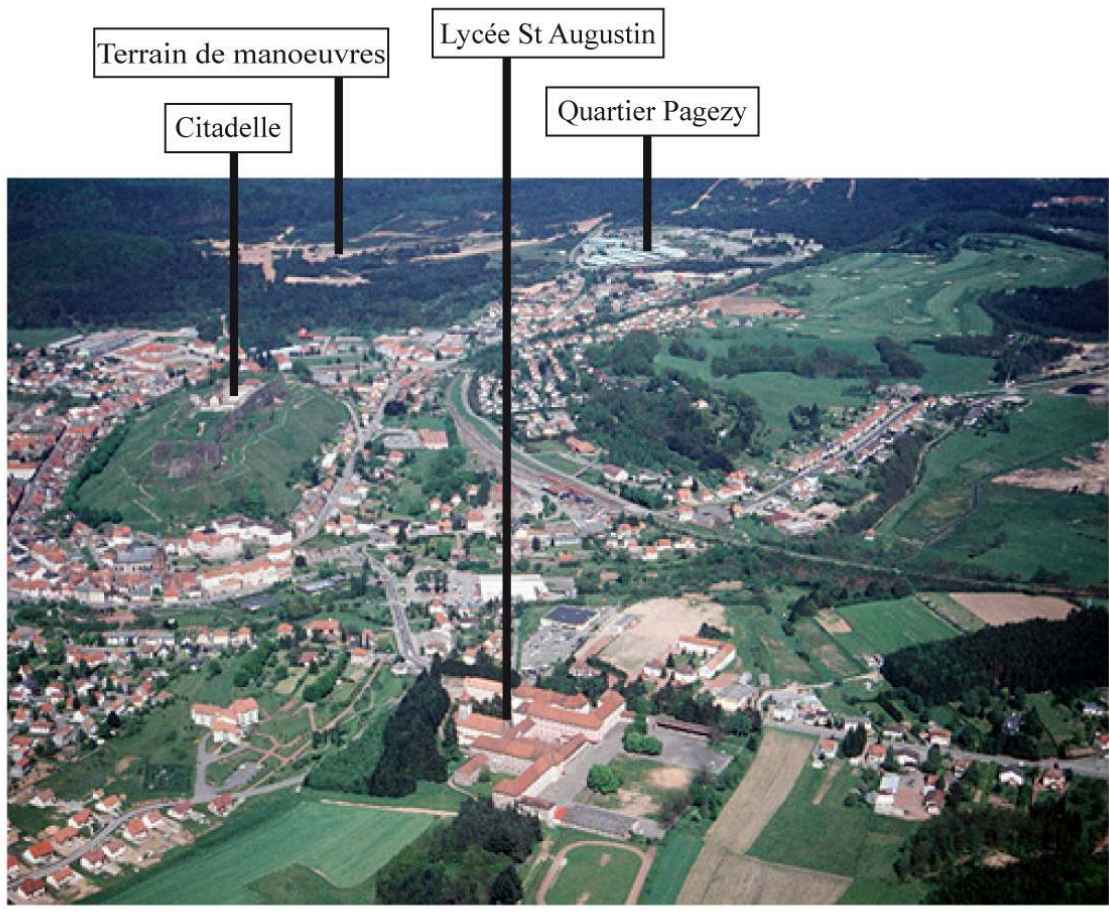

Source: CERPA, 1994

Document $n^{\circ} 3$. Bitche, du centre-ville au camp militaire.

\section{La Meurthe-et-Moselle, moins affectée}

11 Les communes de Nancy, Essey-les-Nancy, Vandoeuvre-les-Nancy et Saulcy-surMeurthe sont concernées : A Nancy, il s'agit du Cercle Mixte, immeuble construit à la fin des années 1970, à Essey, de la caserne Kléber. Les 54 hectares et 86 bâtiments ne sont pas éligibles à l'euro symbolique mais bénéficieront de l'enveloppe globale attribuée au département au titre des PLR. Le projet ARTEM (Art, Technologie, Management), né en 1999, doit permettre à l'Ecole Nationale des Mines, à l'Institut Commercial de Nancy et à l'Ecole Nationale Supérieure d'Art de regrouper leurs activités et leurs 5.000 étudiants sur un site de dix hectares sur le site de l'ancienne caserne Molitor autour du projet d'urbanisme du cabinet d'architecture de Nicolas Michelin, retenu par la Communauté urbaine du Grand Nancy. L'Etablissement Lieutenant Faron de Vandoeuvre bénéficie quant à lui d'une situation de centre ville qui devrait faciliter sa reconversion: la caserne, occupée par l'ESCAT, ferme en 2011. Enfin on citera à Saulcy, les bâtiments du Magasin d'habillement intendant 
général Adrian, qui datent du début des années 1980 et sont implantés sur un terrain de 7 hectares.

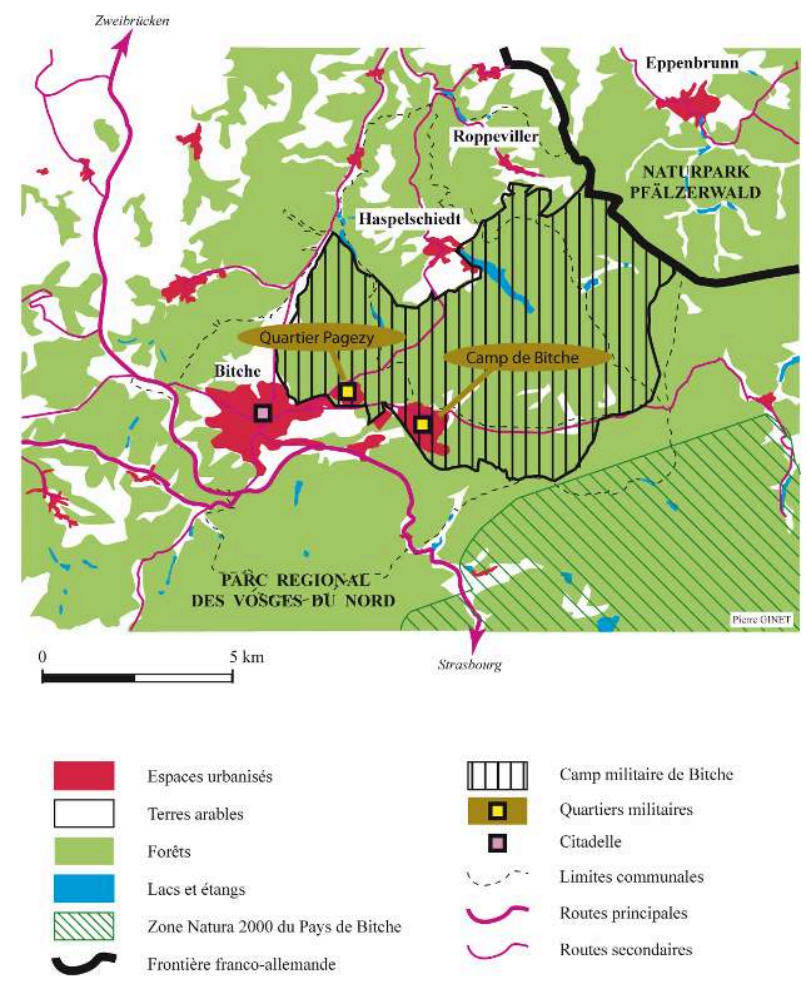

Document $n^{\circ} 4$. Le pays de Bitche, au cœur d'enjeux militaires, écologiques et transfrontaliers.

\section{La Meuse, parent pauvre de la Lorraine}

12 Après l'annonce à l'été 2008 du départ du 8e RA de Commercy, le Président de la République décide que ce dernier n'interviendra qu'après la mise en place de compensations par d'autres activités couvrant les pertes du régiment. Le Ministère de la Défense réaffirme le 14 avril 2010 qu'aucune dissolution n'interviendra tant qu'une solution équilibrée en termes d'emplois ne sera pas trouvée. De nombreux élus tardent à préparer la reconversion. Le bassin d'emploi de Commercy ne recèle pourtant pas de gisement d'emploi public. L'emprise libérée n'offre ni infrastructure d'accueil disponible ni réserve foncière et Commercy souffre d'un fort déficit d'attractivité... Des perspectives de reconversion se limitent à l'idée de création d'une zone franche, à des mesures fiscales et à de prêts destinés à inciter les entreprises à s'installer. La signature d'un contrat de développement économique le 18 juillet 2011 par le préfet de la Meuse et les présidents du conseil régional, des chambres consulaires, du conseil général et de la Communauté de communes du Pays de Commercy dote depuis peu le territoire d'une enveloppe de 25 millions d'euros destinés à soutenir des actions de développement économique et le développement de l'offre de formation dans des domaines d'intérêt local (agroalimentaire, filière bois, tourisme). La fermeture du régiment pourrait également coïncider avec l'ouverture du centre de stockage de déchets radioactifs de Bure, permettant une relance économique dont l'impact régional, tant économique qu'écologique, reste toutefois source de nombreuses inquiétudes ${ }^{1}$. 


\section{B - Le dispositif compensatoire proposé par l'Etat aux territoires concernés par la réforme}

13 Les projets urbains mis en œuvre sur les territoires lorrains concernés par la réforme des Armées (Doc. $\mathrm{n}^{\circ} 5$ ) s'appuient sur un dispositif juridique et financier défini et proposé par l'Etat ainsi que sur la mobilisation d'acteurs très variés dont certains semblent a priori étrangers à ce type de démarche.

\section{Des autorités militaires fortement impliquées dans la restructuration}

14 «L'armée française est en charge de la sécurité des Français, pas de l'aménagement du territoire » (Sarkozy, 2010). Dans son discours aux Armées du 8 janvier 2010, le Chef de l'Etat prononce cette phrase souvent commentée, mais en décalage avec la réalité d'autorités militaires très actives dans la conduite de la politique de restructuration qui suit la fermeture des sites de défense.

15 C'est le cas de la Région Terre Nord-Est (RTNE), qui s'étend du Nord-Pas-de-Calais à la Franche-Comté et englobe les quatre départements lorrains. Cette circonscription de l'Armée de Terre comprend 23 départements regroupant 14 millions d'habitants soit un quart de la superficie et de la population de la métropole et $20 \%$ du Produit Intérieur Brut national. Principal espace de déploiement de la réforme et acteur clef de la mise en œuvre des mesures compensatoires, la RTNE emploie 40.000 militaires et 6.000 civils au sein de 48 établissements militaires. Elle concentre $40 \%$ des restructurations de l'armée de Terre, $42 \%$ des unités fermées et $38 \%$ des unités transférées. Son domaine immobilier est estimé à 700 immeubles, répartis sur 70.000 hectares. Son poids économique atteint quant à lui près de 3 milliards d'euros dont 2,5 en salaires. Ce poids lourd du Ministère de la Défense intervient directement dans l'aménagement du territoire en participant à l'activité diagnostique et programmatique des Comités de site placés sous l'autorité du préfet.

16 D'autres acteurs militaires font aussi de l'aménagement. Le Délégué régional aux restructurations de défense relève ainsi du Ministère de la Défense et travaille en partenariat avec le préfet de Région, sous l'autorité du Délégué Aux Restructurations (DAR) et en liaison avec la DATAR pour suivre les mesures d'accompagnement territoriales destinées à aider les territoires touchés par la réforme. Il étudie les opportunités de réaffectation foncière et immobilière avec la Mission pour la Réalisation des Actifs Immobiliers (MRAI) et traite du reclassement du personnel touché par la réforme avec la Mission d'Accompagnement des Restructurations. La DAR est quant à elle rattachée au Secrétariat Général pour l'Administration (SGA) du Ministère de la Défense. Elle se charge des dossiers nécessitant un accompagnement économique et social. Elle apporte son soutien aux préfets et complète le rôle des DRRD placés auprès des préfets dans les régions les plus touchées par la restructuration.

\section{Outils et modalités d'application des mesures compensatoires}

17 Les CRSD constituent la mesure la plus lourde du dispositif compensatoire. Ils forment le cadre de l'élaboration de projets de développement territorial sur les espaces concernés qui enregistrent une perte supérieure à 200 emplois ou en grande fragilité socio-économique. La procédure s'applique trois ans, reconductible une fois 
pendant deux ans. L'enveloppe budgétaire 2009-2015 est de 225 millions d'euros partagés entre une trentaine de sites.

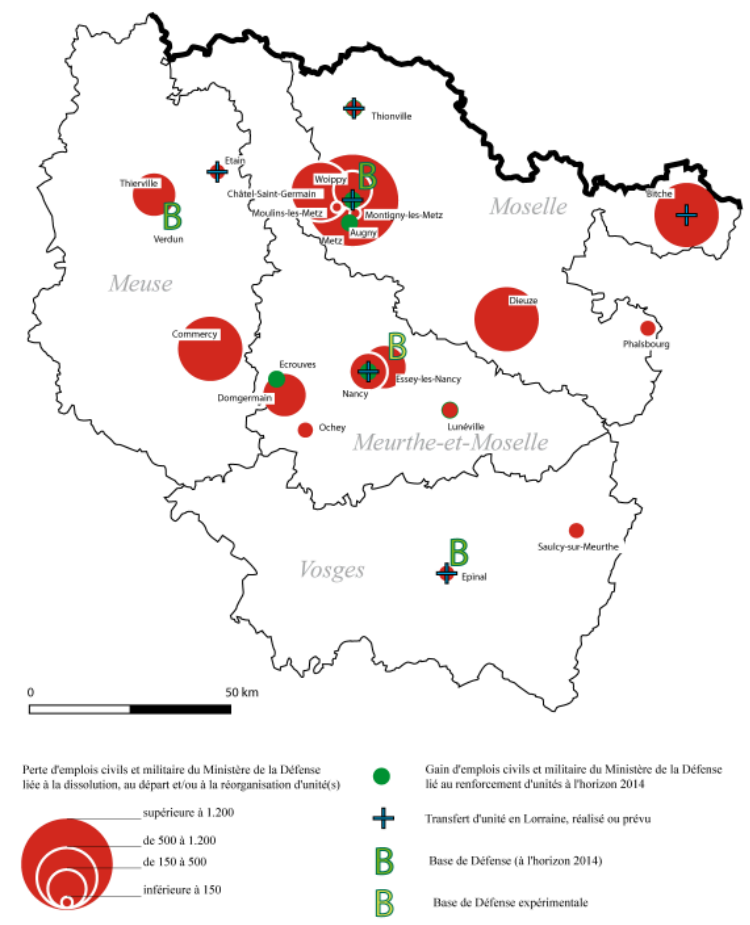

Document $n^{\circ} 5$. Espaces concernés par les restructurations militaires en Lorraine.

18 Les PLR concernent quant à eux les sites moins affectés, enregistrant toutefois un déficit d'emplois compris entre 50 et 200 . Cette procédure déconcentrée mobilise, à l'instar des CRSD, une enveloppe affectée par le préfet de Département après concertation entre collectivités locales et autorités militaires.

19 D'autres dispositifs d'accompagnement de territoires à l'échelon local peuvent également intervenir : les Aides à Finalité Régionale (AFR) destinées à encourager les entreprises à développer des projets de développement ou le Fond d'accompagnement des communes en particulier. Le FReD et le Fond National d'Aménagement et de Développement du Territoire (FNADT) constituent quant à eux la contribution du Ministère de la Défense aux créations d'emplois, à la redynamisation économique et aux projets de réutilisation des sites militaires aliénés. Ces deux derniers fonds peuvent être mobilisés conjointement dans le périmètre des CRSD et des PLR.

20 Comme le prévoit l'article 67 de la loi de Finances 2009, certains immeubles domaniaux dont le Ministre de la Défense souhaite se défaire peuvent prétendre être cédés pour un euro symbolique. Mais ces cessions doivent déboucher sur un partage des bénéfices après réalisation d'un aménagement par la collectivité et lors de la revente du bien dans un délai de 15 ans après acquisition. En cas d'absence de revente ou de cession des biens pendant ce délai et en l'absence d'opération d'aménagement, l'Etat peut cependant racheter l'immeuble à l'euro symbolique. Il ne s'agit donc pas d'un cadeau. Par ailleurs, toutes les communes ne sont pas éligibles à cette politique de redynamisation de sites après la perte d'effectifs de militaires. Elles doivent connaitre une situation de grande fragilité économique, 
sociale et démographique et avoir peu ou pas de capacité à impulser une dynamique de développement sans intervention de l'Etat. La liste de ces communes - une centaine dans 26 départements - est fixée par un décret de juillet 2009.

Le Plan Grand Nord-Est présente enfin la particularité d'être l'unique dispositif de relance d'échelon régional. Il est - ou plutôt était - destiné à participer «au développement durable et à l'attractivité du Nord est de la France » (Ministère de l'écologie, de l'énergie, du développement durable et de l'aménagement du territoire, 2008). Sous la gouverne du Préfet H.Blanc, le pilotage de ce dispositif transversal particulièrement ambitieux reste assuré par les préfets de Départements ou de Régions en coordination avec les acteurs locaux sur une période de trois à cinq ans.

\section{Procédure de mise en place des mesures compensatoires}

22 Des Comités de site, dirigés par les préfets, ont été mis en place. Leur mission : faciliter le dialogue entre les différents acteurs et permettre l'élaboration de diagnostics territoriaux, la définition d'axes stratégiques prioritaires et l'établissement de plans de restructuration. 23 sites ont fait l'objet d'un diagnostic entre 2008 et 2009. 60 nouveaux diagnostics ont été engagés sur la période 2009-2014. Les trois impératifs sont la revitalisation territoriale, le reclassement des personnels et la reconversion territoriale. Suit le plus souvent une phase de dépollution des sites, obligatoire avant toute cession à un tiers (Grasmück, 2008). Celle-ci peut être prise en charge par l'acquéreur lors du processus de cession à l'euro symbolique. Trois types de dépollution peuvent être distinguées : Obligatoire (amiante, hydrocarbures, plomb): les études sont réalisées par le Service d'Infrastructure de la Défense; Pyrotechnique : le Ministère de la Défense apporte les éléments relatif à l'histoire du site et au diagnostic avant dépollution; Enfin lorsque des installations classées pour la protection de l'environnement ne sont pas reprises par l'acquéreur, l'exploitant a l'obligation de remettre en état le site lors de leur arrêt définitif. On notera enfin que la phase de gardiennage, très coûteuse, concerne les sites qui, n'étant pas cédés à un tiers, restent sous la responsabilité de l'autorité militaire : une contrainte qui incite a priori à leur revente rapide.

\section{II - Restructurations militaires : quelle place pour la Lorraine sur l'échiquier de l'aménagement du territoire national ?}

23 Les effets des mesures de restructuration qui succèdent à la réforme des Armées apparaissent efficients et efficaces si l'on se borne à observer au cas par cas les démarches de projets urbains et de territoires menées à l'échelon local. Toutefois, « dans nos sociétés technocratiques et centralisées, contester les experts du pouvoir et ébranler leurs certitudes sont une quasi nécessité dans la mesure où c'est souvent la seule façon de (...) mettre en avant d'autres choix d'aménagement et de développement » (Hulbert, 2006). Replacées à l'échelon régional et national, outre l'action finement orchestrée de l'Etat, c'est la logique fragmentaire d'acteurs locaux qui se révèle. Cette dernière affaiblit la région dans sa globalité, une région lorraine dont le manque d'épaisseur territoriale et d'affirmation identitaire à cet échelon, 
ouvre des perspectives au déploiement d'une logique d'Etat dans laquelle ses acteurs, à la fois trop nombreux et trop divisés, n'ont pas la garantie de tirer collectivement leur épingle du jeu.

\section{A - Des acteurs locaux sous perfusion}

Ainsi font, font, font...

24 L'articulation des échelons décisionnels du local au national, au niveau territorial (collectivités locales, préfectures, chambres consulaires, associations...) et entre les ministères concernés, définit les termes et les conditions du succès de la mise en œuvre des mesures compensatoires. La mobilisation d'acteurs nombreux et diversifiés apparaît a priori bénéfique pour les territoires car garant de créativité et de dynamisme. La présence d'acteurs locaux porteurs de projets (communes, communautés de communes) et d'acteurs coordinateurs au niveau de l'Etat (DIACT, préfet de Départements, superpréfet du Plan Grand Nord-Est) place toutefois ce dernier en position centrale. Au plan national, la décision des grandes lignes à suivre ainsi que l'attribution de tâches par le Premier Ministre dans sa circulaire du 28 juillet 2008 définit les termes d'une coopération diligentée par l'Etat. Sa traduction à l'échelon local prend ainsi la forme de la constitution, par les préfets de Départements, de Comités de sites de défense déjà évoqués, qui regroupent l'ensemble des acteurs locaux concernés par un CRSD. L'offre de moyens a coïncidé avec une soudaine volonté du gouvernement de mettre à la disposition des acteurs locaux un large panel de prestataires techniques nationaux (DIACT, DAR, MRAI, France Domaine, AFII...), d'un milliard d'euros (au plan national), de mesures et de textes de loi spécifiques. Elle se traduit par des déplacements d'H.Falco le 28 juillet 2008 à Bitche, le 14 octobre de la même année à Metz, puis d'H.Blanc le 13 octobre 2008 et même du Président de la République lors d'une table ronde le 8 octobre 2009 à Woippy, un mois après que ce dernier ait reçu quelques élus mosellans à l'Elysée.

25 Après le bâton, la carotte. La mise à disposition par l'Etat d'un kit de survie pour région en crise, une fois la Réforme annoncée, assoit son contrôle sur les démarches de terrain. Dès 2009, l'officialisation des ZRD, l'extension des AFR, la création d'un pôle INSEE, marquent le démarrage de la phase de projets. Très vite, certains territoires lorrains apparaissent quelque peu délaissés. Les visites d'Etat ont jusqu'à présent surtout concerné la Moselle. Concernant la Meurthe-et-Moselle et la Meuse, il est bien difficile de trouver date d'une visite d'H.Falco, d'H.Blanc ou d'autres représentants de l'Etat. Au final, l'Etat joue son rôle d'impulsion d'idées, de pourvoyeur de crédits tandis que les élus locaux se coulent dans le moule confortable de leur principal partenaire, fournisseur d'un livre de recettes prenant la forme de méthodes et d'outils d'aménagement et de développement territorial qu'ils s'emploient à utiliser dans le périmètre de leurs circonscriptions respectives.

\section{Chacun pour soi... et l'Etat pour tous}

26 L'annonce de la réforme par l'Etat a généré un bref mouvement de contestation. L'échelon local a alors manifesté son unité au travers de manifestations d'élus (6 septembre 2008 à Metz), de menaces de démission, de propos virulents et plus généralement de formes éphémères et quasi obligées de rassemblement. Mais 
l'importance des conséquences de la réforme en Moselle et plus particulièrement à Metz jette une ombre sur les autres sites touchés. La coopération et les groupes de réflexion locaux tardent à se former tandis que les conflits politiques contribuent à enliser un peu plus les territoires (cf. les relations exécrables entre la Ville de Verdun et le Conseil général de la Meuse par exemple). Partout et à l'instar des grands dossiers lorrains (saupoudrage d'éoliennes en Meuse, implantation du site nucléaire de Bure...), le citoyen apparaît trop éloigné du débat, à l'écart d'une coalition d'intérêt entre décideurs politiques locaux et nationaux, seuls véritables bénéficiaires, respectivement d'un point de vue électoral et au regard de logiques nationales d'aménagement du territoire, alors même que son espace de vie se trouve profondément bouleversé, voire menacé. Outre les retards pris par la signature des plans d'aide de l'Etat et leur engagement concret (le CRSD de Metz a vu sa signature décalée de plus d'un an et demi), c'est la capacité à formuler des idées nouvelles des acteurs de terrain qui est en cause. Mais surtout, si chaque site touché parvient à rassembler un maximum d'acteurs locaux, on peut noter une absence d'échanges directs, sans même parler de mise en réseau de tous ces sites. Outre leur incapacité à faire émerger des démarches endogènes a fortiori synergiques, les acteurs politiques locaux renforcent la concurrence des territoires en matière d'attraction de capitaux, d'entreprises et de projets. Chaque euro utilisé fini en effet par s'intégrer dans un projet structurant un espace d'échelon exclusivement infra-régional (au mieux départemental pour les PLR et au niveau des bassins d'emplois pour les CRSD), contribuant in fine à scléroser ou à obscurcir un peu plus le puzzle géopolitique lorrain.

27 Mais le point noir est sans conteste le Plan Grand Nord-est (Sénat, 2010). Son échec et le silence qui règne autour de lui apparaissent d'autant plus tonitruants que les attentes régionales étaient importantes. Le Conseil Régional de Lorraine s'y est investi dans un rapport du 8 décembre 2008 dans lequel il fixait des orientations stratégiques pour l'espace lorrain dans son ensemble, reprenant les recommandations d'H.Blanc, et proposant des actions concrètes en terme de développement des réseaux ferrés, routiers, fluviaux, de développement universitaire, touristique... Après une annonce médiatisée et les visites d'H.Blanc sur les sites militaires, cette mission est désormais au point mort: absence de crédits, de déplacement ou de déclaration d'H.Blanc durant 2009, rapport censé être rendu public en mars 2009 mais jamais produit...

28 A l'Est, rien de nouveau. Le Plan Grand Nord-Est révèle finalement les clivages dont la lorraine ne parvient pas à sortir même lorsqu'elle est confrontée aux crises les plus fortes. Les querelles demeurent prépondérantes: l'envoi d'une délégation mosellane et non lorraine à Paris, lors de la rencontre avec le Chef de l'Etat le 2 septembre 2008 en est une illustration édifiante. La mission Grand Nord-Est et l'investissement attendu du Conseil régional de Lorraine, trop en retrait, voire impuissant, constituent pourtant les conditions indispensables pour voir émerger une dynamique de développement d'échelon régional puissante. Mais l'Etat apparaît ici comme le seul ciment des acteurs locaux et impose sa volonté dans le sens qu'il souhaite en jouant sur les discordances politiques intra-régionales tout en apparaissant à l'échelon local comme principal fournisseur d'aides au redéveloppement. 


\section{B - Une vision globale et ascendante trop timide}

\section{Les think tanks lorrains : l'exemple de l'engagement de l'AR13}

29 Une réflexion a été menée durant la période 2008-2009 par un comité composé d'auditeurs de l'association lorraine de l'institut des hautes études de défense nationale (AR13), preuve que des cercles de réflexion actifs existent en Lorraine. La posture de ce groupe détonne dans le paysage lorrain car elle envisage les restructurations militaires comme une opportunité de voir les acteurs locaux se rassembler... enfin! «Cet électrochoc peut permettre aux acteurs de la région de rompre définitivement avec l'attentisme, la division et l'opportunisme pour s'engager dans un mode de fonctionnement volontariste et intégrateur » (Bailly et alii, 2009).

\section{L'idée d'un « Carrefour européen des Cultures » articulé sur une « Transversale Européenne de la Paix»}

30 Le Comité propose la mise en œuvre d'un grand projet d'aménagement et de développement fédérateur, à base culturelle forte et prenant appui sur les orientations stratégiques majeures préexistantes. Il entend valoriser la volonté du Conseil Régional de Lorraine de «créer un réseau culturel à vocation européenne autour du tourisme patrimonial en Lorraine» (Conseil Régional de Lorraine, 2008) et souligne le contexte historique favorable à la valorisation du patrimoine militaire (commémoration du 90ème anniversaire de l'Armistice à Verdun, classement au Patrimoine mondial de l'UNESCO des sites Vauban). « Une telle posture, qu'autorise la Réforme des armées, permettrait à la Lorraine de renouer avec une histoire (...), d'affermir l'image qu'elle renvoie tant auprès de sa propre population et de ses décideurs qu'à l'échelon national et transfrontalier, et de consolider sa cohésion politique. Ce projet doit constituer une locomotive dont le pilotage conjoint par Metz et Nancy, pôles majeurs de la région, constituerait un pas dans la mise en cohérence de la gouvernance des territoires régionaux » (Bailly et alii, 2009).

31 La répartition géographique des sites à valoriser en Lorraine est inégale (Cassagne, Rufflet, 2007) : sites "de mémoire", ancrés dans le passé : cimetières, champs de bataille, zones rouges, lieux d'histoires figés dans une intemporalité propice devant permettre le recueillement et la réconciliation; Sites "actifs », casernes, espaces militaires; Sites "pédagogiques ", tournés vers le futur et à même de prévenir en informant les plus jeunes, l'éclosion de nouveaux conflits. Leur valorisation économique et territoriale par le tourisme militaire pourrait prendre la forme d'une «route européenne de la paix et de la mémoire» (Bailly et alii, 2009). Un projet envisagé à l'échelle d'une Lorraine élargie à l'ensemble de la Grande Région. Cette Transversale, en rassemblant les sites, permettrait de renouveler le concept de mémoire, désormais sans écho auprès des jeunes. «Un signal fort d'un changement d'échelle possible, plaçant Verdun au rang mondial des symboles de la paix au même titre qu'Hiroshima ou Nagasaki, et générateur d'une ouverture symbolique sur les voisins européens. Un projet pour lequel la Lorraine, renouant avec son identité menacée de délitement, jouerait un rôle d'entraînement au plan national et mobiliserait plus facilement les fonds européens à même d'asseoir sa position une fois le processus de développement enclenché. La mise en réseau de ces lieux de 
Mémoire pourrait être valorisée par une animation structurée à la fois autour d'une gestion quotidienne, de rendez-vous réguliers et de grands événements : un Forum annuel mondial de la Paix au Centre Mondial de la Paix de Verdun, sous la présidence du Secrétaire Général de l'Organisation des Nations Unies, dressant épisodiquement le bilan mondial des actions ayant permis le maintien de la paix, le retour à la paix » (Bailly et alii, 2009). L'ambition et les idées ne manquent pas... mais leur déclinaison politique se heurte aux luttes incessantes de Clochemerle à la conquête de parcelles de pouvoir.

\section{C - Sans démarche régionale, une Lorraine dépecée par l'Etat ? Des signes avant coureurs...}

32 Les réflexions de l'AR13 sont très loin d'avoir porté leurs fruits. La capacité de la Lorraine à faire émerger une dynamique endogène puissante d'échelon régional demeure à ce jour illusoire. Les luttes de pouvoir internes prévalent et ouvrent le terrain, en l'affaiblissant, aux stratégies de l'Etat comme en témoignent les modalités de mise en œuvre de la politique nationale de soutien à la métropolisation ou les conditions de localisation de grands équipements au potentiel de nuisance important.

\section{Diviser pour régner ?...}

33 La conurbation transfrontalière ou Métropole Sarrebruck/Moselle-Est (MSME) est ainsi l'une des aires urbaines retenues en 2003 au titre de l'Appel à coopération métropolitaine lancé par l'État. Celui-ci entend une fois encore soutenir le rayonnement métropolitain. La MSME forme une conurbation transfrontalière qui s'étend de part et d'autre de la frontière franco-allemande, du Nord-Est du département de la Moselle au Sud du Land de Sarre. Ce territoire de 660.000 habitants, centré sur Sarrebruck, est marqué par une longue histoire économique et sociale commune, et confronté à l'enjeu de la reconversion de son bassin industriel. Le projet métropolitain doit faciliter l'instauration d'une coopération durable entre les communes et les structures intercommunales de part et d'autre de la frontière et permettre d'affirmer l'existence d'une métropole de gabarit européen. En acceptant la candidature d'une MSME absente des grandes aires urbaines françaises, dépourvue d'une identité territoriale forte, multipolaire et diluée, l'Etat donne un coup de pouce qui s'apparente de prime abord à une dernière chance offerte à un territoire bien malmené par l'histoire économique, politique et sociale des dernières décennies. Mais la Lorraine avait-elle besoin de deux métropoles ? La région semble timidement sortir de l'antagonisme Metz-Nancy. Le volet "Sillon Lorrain" de l'Appel à coopération va dans ce sens, de même que la création en cours de l'Université de Lorraine. Un rattachement de la MSME au Sillon lorrain aurait permis de conforter cette dynamique. L'Etat aurait pu ainsi impulser un réseau de villes, à l'instar de la Métropole Normande, de la métropole Rhin-Rhône, de Clermont Auvergne Métropole ou de la Région Urbaine de Lyon... Encore eût-il fallu que les acteurs lorrains concernés le souhaitent. L'introduction d'une métropole-bis est par contre génératrice d'une nouvelle division, à la fois inutile et dangereuse pour la Lorraine. Avec moins de 700.000 habitants, seules de rares agglomérations telles que Luxembourg parviennent à s'inscrire dans le concert des métropoles. Le 
choix du gouvernement de retenir une MSEM taillée si petite au lieu d'une grande métropole lorraine l'associant au "Sillon lorrain » détonne d'ailleurs avec le constat exprimé par le Délégué de la DATAR : « La (...) motivation du gouvernement vient du constat de relative modestie des métropoles françaises, quand on les compare à leurs voisines européennes ou internationales " (Jacquet, 2004). Mais, comme nous l'avons vu précédemment, l'Etat n'est pas à une contradiction ou à un revirement prêt dans ce jeu du chat et de la souris.

\section{...en prenant appui sur le jeu ambigu des collectivités locales?}

34 La scission entérinée par ce choix, en affaiblissant chacun de ses deux bénéficiaires et par conséquent la Lorraine dans son ensemble, profite aux pôles métropolitains " confirmés » du voisinage (Strasbourg, Paris, Luxembourg). Le soutien apporté à la MSME au détriment d'une grande et unique métropole lorraine fragilise sans le dire cette région, et favorise in fine un changement d'échelle du développement du territoire national. Cette mutation visible dans l'affirmation de l'axe ParisStrasbourg ${ }^{2}$, laisse pour compte l'espace interstitiel constitué par la marge lorraine, avatar toujours prégnant de l'ancienne Lotharingie. Restera à l'Etat dans quelques années à secourir la Lorraine à l'aide de grands projets difficiles à faire admettre ailleurs. Pourquoi ne pas laisser par exemple l'Agence Nationale pour la gestion des Déchets Radioactifs (ANDRA) faire bénéficier la Lorraine de ses retombées... financières en légitimant une vocation de poubelle nucléaire nationale ou mieux, européenne? Un scénario qui ne fonctionnerait pas sans la collaboration active et l'opportunisme d'acteurs locaux.

\section{Conclusion}

35 Hormis la création de bases de défense ${ }^{3}$, l'impact de la réforme des Armées sur la Lorraine se traduit par la dévitalisation de sites militaires puis par la mise à la disposition des collectivités locales d'outils destinés à leur permettre de replacer les personnels, impulser de nouvelles dynamiques de projet, de revitaliser les territoires et finalement de permettre la conversion des espaces concernés. La géographie des sites touchés par la réforme répond à une répartition aréolaire avec une concentration privilégiée dans l'agglomération messine, puis en Moselle et enfin dans les autres départements. A ce gradient décroissant du centre vers la périphérie lorraine s'ajoute le fait que les sites sont principalement situés en ville ou à proximité d'agglomérations. Cette répartition, en plaçant la capitale régionale en position centrale, aurait pu constituer pour l'Etat une opportunité extraordinaire de soutenir une démarche de développement régional des espaces en mutation dans le cadre d'une mise en réseau d'actions placées sous la gouverne nancéio-messine. Pourtant l'appui de l'Etat relève plutôt d'un saupoudrage, et prend la forme d'aides focalisées sur des dynamiques locales. L'appui d'échelon régional, via le Plan Grand Nord-Est est un échec. Quant aux démarches endogènes de développement régional, elles demeurent au mieux à l'état de réflexion. Par conséquent, le succès apparent des démarches ponctuelles de développement liées aux restructurations ne doit pas faire illusion. Profitant de l'incapacité de nombreux acteurs locaux lorrains à produire un projet de développement collectif, la réforme de la carte militaire et surtout les modalités de mise en œuvre de la restructuration qui la suit, constituent un vecteur parmi d'autres d'un projet d'aménagement du territoire national dans 
lequel elle se réduit à n'être plus qu'un espace de marge. N'oublions pas que les réflexions intervenues au cours du projet Balladur sur la réforme des collectivités territoriales ont été jusqu'à proposer un dépeçage de la Lorraine, ses départements étant répartis entre les régions voisines! De même, le Comité pour la réforme des collectivités locales, dans sa proposition $n^{\circ} 8$ a bien retenu l'idée de « créer par la loi onze premières métropoles, à compter de 2014 " (Comité pour la réforme des collectivités locales, 2010), sans que ni Metz ni Nancy n'apparaissent dans ce peloton de tête, contrairement aux villes voisines de Strasbourg, Lyon ou Lille... De là à dire que la Lorraine, région d'entre-deux depuis l'an 843, n'existe pas, il n'y a qu'un pas. De fait, ses acteurs politiques sont aujourd'hui encore incapables de faire émerger une stratégie de développement collective puissante, à l'instar de la région Nord-Pas-de-Calais, région sœur qui a elle, réussi à surmonter ses dissensions en se rassemblant autour d'un projet d'avenir fédérateur dès les années 1990 (Ginet, 2010). En 2005, la décision de l'Etat d'offrir à la Lorraine deux métropoles (Sillon lorrain et Métropole Sarrebruck Moselle est) au lieu de la contraindre à concentrer ses efforts sur une seule région métropolitaine n'était-elle pas déjà un moyen d'entériner voire d'entretenir cette situation? Cet appui sélectif de l'Etat à la métropolisation semble traduire le choix d'un territoire national du futur dans laquelle la Lorraine déjà réduite à choisir les dynamiques de développement les plus impopulaires (poubelle nucléaire), serait sacrifiée. Autrement dit, vu de Paris, plutôt Strasbourg que Metz-Nancy. Pourtant les perspectives d'un développement endogène existent. L'échéance du centenaire de la Grande guerre offre aux acteurs lorrains l'occasion de se mobiliser plus que certains ne le font déjà, pour créer autour d'un grand événementiel les conditions d'un nouveau développement et de rattraper leur retard sur d'autres espaces (Normandie) dans la valorisation concurrentielle d'une offre mémorielle et touristique en partie comparables. Les réflexions menées au sein de think tanks lorrains, bien que non emparées par les décideurs politiques laissent encore subsister un espoir en attestant d'une capacité régionale à mener ce type de réflexion transversale.

\section{BIBLIOGRAPHIE}

Bailly J-P., Bitsch D., Douillot S., Ducloux P., Dupuis-Rémond J-C., Ginet P., Houver M., Husson J-P., Lagniez J., Lahalle-Gravier P., Malhomme M-C., Manciet E., Mentré G., Schwarz-Koehler S., Wingerter J., 2009, Les restructurations militaires en Lorraine: Opportunité pour un nouveau projet fédérateur dynamique, garant du lien armée nation et d'une culture de défense, Metz, Association Lorraine de Institut des Hautes Etudes de Défense Nationale, 20p.

Barbier M., 2010, Le devenir des emprises aliénées par la Défense sur le territoire de la Région Terre NordEst, Mémoire de Master DEMETERR (Défense - Méthodes - Territoires), Université de Metz.

Brunet R., Ferras R., Thery H., 1993, Les mots de la géographie, dictionnaire critique, Paris, Reclus-La Documentation française. 
Cassagne A., Rufflet M., 2007, Le lien armée-nation. Historique et perspective, Montpellier, Premières armes, $148 p$.

Comité pour la réforme des collectivités locales, 2010, Les vingt propositions.

Commission du Livre blanc sur la défense et la sécurité nationale, 2008, Défense et Sécurité nationale. Le Livre blanc, Paris, Odile Jacob / La documentation française, juin, 415p.

Conseil Economique et Social de Lorraine, 2008, Les Armées en Lorraine. D'une histoire en devenir à une histoire sans avenir?, Metz, Rapport d'étude.

Conseil Régional de Lorraine, 2008, Le plan de développement durable et d'attractivité du Grand NordEst. Les priorités du Conseil Régional, Metz, Séance plénière du 19 décembre.

Est Républicain - 20 octobre 1993 - « Le laboratoire souterrain, une chance à saisir pour la HauteMarne ».

Ginet P., 2010, La métropole lilloise au tournant des années 1990. L'histoire exemplaire d'une renaissance urbaine, Saarbrücken, Editions universitaires européennes, 334p.

Grasmück M., 2008, La gestion de la pollution par le ministère de la Défense des terrains qui lui sont affectés, Mémoire de Master, Université de Metz, 216p.

Hulbert F., 2006, «Luttes urbaines et engagement citoyen : la construction d'une géopolitique dans l'action », in Hulbert F., Villes du Nord, Villes du Sud, géopolitique urbaine, acteurs et enjeux, Paris, L'Harmattan, texte 57, chapitre VII, p579-585.

Jacquet N., 2004, « Interview de Nicolas Jacquet, Délégué à l'aménagement du territoire et à l'action régionale », Les dossiers FNAU, $n^{\circ} 15, \mathrm{p} 7$.

Journal Officiel du Sénat du 14 janvier 2010 - Question orale sans débat n0761S de Yves Daudigny, Sénateur socialiste de l'Aisne.

Kubiak Y., Serre O., 2010, «Evaluation de l'impact des restructurations militaires sur l'espace résidentiel de l'agglomération messine », INSEE Lorraine, $\mathrm{n}^{\circ} 215$.

Kubiak Y., Serre O., 2009, « Départ du 13ème RDP : Un impact géographique très localisé », INSEE Lorraine, $\mathrm{n}^{\circ} 186$.

Ministère de l'écologie, de l'énergie, du développement durable et de l'aménagement du territoire, Restructurations militaires : Hubert Falco dresse un premier bilan de ses déplacements et installe Hubert Blanc dans la mission grand Nord-est, Conférence de presse, 16 septembre 2008, 30p.

Sarkozy N., Allocution du Président de la République, Vannes, 8 janvier 2010.

\section{NOTES}

1. Stopbure.com, www.stopbure.com, 15 juin 2007.

2. Cf. la délocalisation de l'Ecole Nationale d'Administration de Paris à Strasbourg conjointe à la mise en place du TGV-Est.

3. La Base de Défense devient l'unique formation administrative de la Défense à l'échelon local. Elle est constituée par le rassemblement de forces à dans un espace géographique d'un rayon d'environ $30 \mathrm{~km}$ ou d'1h30 de trajet. 87 BDD, opérationnelles d'ici 2014, seront constituées. Elles permettront de rationnaliser le soutien et de faire des économies sur le personnel en permettant une diminution des effectifs. 


\section{RÉSUMÉS}

Le Livre Blanc sur la défense et la sécurité nationale, paru en 2008, conduit à la disparition de nombreuses unités militaires en Lorraine. Un choc de plus pour cette région, contrebalancé toutefois par des mesures compensatoires qui conduisent les acteurs politiques locaux à s'engager dans des projets de redéveloppement. Cette dynamique de réaménagement du territoire lorrain n'est cependant pas un jeu à somme nulle. La logique de guichet d'un trop grand nombre d'acteurs politiques locaux et leur manque de capacité à faire émerger une stratégie de rebond fondée sur la mise en œuvre d'une démarche d'échelon régional, accentuent les clivages territoriaux. Cette configuration géopolitique et cette mise en perspective géostratégique facilitent la mise en œuvre par l'Etat de projets d'aménagement d'intérêt national dans lesquels l'avenir de la Lorraine apparaît bien sombre.

The white paper upon defence and national security published in 2008, leads to the loss of many army settlements in Lorraine. A new traumatism for this region however offset with compensating measures which bring local political actors to launch new development projects. But this way of a new Lorraine territorial planning is not a null summation play. The ticket office habit of a large number of local political actors and their lack of capacity to start up a bounce strategy built up on a regional process, increase territorial splits. This geopolitical and geostrategic shape makes finally easier the state implementation of national interest planning projects in which the future of the Lorraine region seems gloomy.

\section{INDEX}

Mots-clés : restructurations, défense, aménagement, géostratégie, prospective, Lorraine Keywords : territorial restructuring, defence, planning, geostrategy, forecasting, Lorraine

\section{AUTEUR}

\section{PIERRE GINET}

CEGUM (EA1105)Université Paul Verlaine - Metz 